\title{
Industrial physicians and orthopedists taking care of sonographers with work-related musculoskeletal disorders
}

\author{
Hiroshi Nakajima ${ }^{1}$
}

(C) The Japan Society of Ultrasonics in Medicine 2015

I serve as the area editor in the field of orthopedics. I appreciate your enthusiastic submissions in English. Submissions cover a wide variety of fields such as injury, tumor, and inflammation of limbs and spine. An area with few submissions is labor hygiene. An article pertaining to labor hygiene was submitted to Volume 42 Issue 4 by a group of engineers [1], but there have been few such submissions from industrial physicians and orthopedists. I welcome manuscripts about work-related musculoskeletal disorders suffered by sonographers.

\section{Reference}

1. Suzuki H, et al. Effect of horizontal console position on operator muscular stress during cardiac ultrasonic diagnosis. J Med Ultrason. 2015;42:499-504.

Hiroshi Nakajima

makitsume@marianna-u.ac.jp

1 Department of Orthopedic Surgery, St. Marianna University

School of Medicine, Kawasaki, Kanagawa, Japan 